\title{
Jensen Type Inequality for the Bipolar Shilkret, Sugeno and Choquet Integrals
}

\author{
Biljana Mihailović $^{a}$, Mirjana Štrboja ${ }^{b}$, Miloš Todorov $^{c}$ \\ ${ }^{a}$ University of Novi Sad, Faculty of Technical Sciences, \\ Trg Dositeja Obradovića 6, 21000 Novi Sad, Serbia, lica@uns.ac.rs \\ ${ }^{b}$ University of Novi Sad, Faculty of Sciences, Department of Mathematics and \\ Informatics, Trg Dositeja Obradovića 4, 21000 Novi Sad, Serbia, \\ mirjana.strboja@dmi.uns.ac.rs \\ c University of Novi Sad, Faculty of Technical Sciences, Trg Dositeja \\ Obradovića 6, 21000 Novi Sad, Serbia, todorov.dt6.2013@uns.ac.rs
}

Abstract: In this paper we prove the Jensen type inequality for the discrete bipolar Shilkret
and Sugeno integrals. We propose the conditions for the validity of this type inequality for
the discrete bipolar Choquet integral. In order to illustrate the obtained results, some
examples are given.

Keywords: bi-capacity; bipolar Choquet integral; bipolar Shilkret integral; bipolar Sugeno integral; Jensen's inequality

\section{Introduction}

Non-additive (fuzzy) integrals with respect to capacities, or fuzzy measures have been studied in $[6,9,14,19]$ as aggregation tools in multicriteria decision analysis, with a wide range of applications in economics, machine learning, engineering, etc. Among these integrals based on capacities, let us mention prominent ones as the Choquet integral [4, 5, 18], the Sugeno integral [26, 27], and the Shilkret integral [22].

In recent years, the concept of bipolar fuzzy integrals more general than integral models such as Cumulative prospect theory (CPT) [31] has been considered in $[8,10-12,16]$. In [11] the discrete bipolar universal integral based on bi-capacities was defined and discussed, as an extension of the bipolar Choquet integration proposed in [8] and the bipolar fuzzy integration introduced in [12]. Recently, the idea was further developed in $[25,28,30]$ by introducing and investigating the bipolar pseudo-integrals and bipolar pan-integrals in discrete settings. 
Due to the integrals based on capacities are used extensively, the research of integral inequalities related to fuzzy integrals based on capacities has been explored by various authors in [1, 2, 13, 15, 20, 21, 24]. Jensen's inequality has usage in many disciplines, such as mathematical economics, probability and optimization theory, etc. This inequality has also been studied for the bipolar pseudo-integral [30]. However, in the case of bipolar pseudo-integrals, the underlining bi-capacities have to be $\bigoplus$-decomposable, where $\bigoplus$ is a corresponding pseudo-addition. Therefore, it is useful to consider other types of integrals, based on the bipolar scale and related to an arbitrary normalized bi-capacity, and to investigate the inequalities of such type for these integrals. Hence, our main goal now is to propose the conditions for validity of the Jensen type inequality for the bipolar Shilkret, Sugeno and Choquet integrals.

This paper is based on [29] and [17]. The paper is organized as follows. In Section 2 , an overview related to bi-capacities and the bipolar fuzzy integrals with respect to bi-capacities is given. In Section 3, the Jensen type inequality for the bipolar Shilkret integral and the bipolar Sugeno integral are proved, based on [29]. Novel results related to the reversed Jensen type inequality are shown and new illustrative examples are given. In Section 4, using results from [17], as a novel result the Jensen type inequality for the bipolar Choquet integral is considered and the reversed Jensen type inequality for this integral is obtained. Finally, some concluding remarks are given.

\section{Preliminaries}

According to $[7,8,11,12]$, in this section, some basic notions related to bi-capacities and the bipolar Shilkret, Sugeno and Choquet integrals are presented. Recall that the symmetric maximum and the symmetric minimum are operations $\mathbb{V}, \mathbb{A}:[-1,1]^{2} \rightarrow[-1,1]$ given by:

$$
\begin{gathered}
x \mathbb{V} y=\operatorname{sign}(x+y)(|x| \vee|y|) ; \\
x \mathbb{A} y=\operatorname{sign}(x \cdot y)(|x| \wedge|y|) .
\end{gathered}
$$

The symmetric maximum $\mathbb{V}$ is not associative, hence we adopt the next convention based on the splitting rule:

$$
\mathbb{V} x_{x_{i} \in I} x_{i}=\sup _{x_{i} \geqslant 0} x_{i} \mathbb{V} \inf _{x_{i}<0} x_{i}
$$

for any subset $I$ of the interval $[-1,1]$.

Let $X=\left\{x_{1}, x_{2}, \ldots, x_{n}\right\}$ be a non-empty set, which defines the set of arguments, and $\mathcal{P}(X)$ be its partitive set.

Recall, a mapping $\mathrm{m:} \mathcal{P}(X) \rightarrow[0,1]$ is said to be a capacity (or fuzzy measure) if $\mathrm{m}(\varnothing)=0$ and for all $A \subset B \subset X$ it holds $\mathrm{m}(A) \leq \mathrm{m}(B)$. 
Definition 1 ([7])A mapping $\mathbf{m}: Q(X) \rightarrow \mathbb{R}$, where

$$
\mathcal{Q}(X):=\{(A, B) \in \mathcal{P}(X) \times \mathcal{P}(X) \mid A \cap B=\emptyset\},
$$

is said to be a bi-capacity if $\boldsymbol{m}(\varnothing, \emptyset)=0$ and for all $A \subset B \subset X$ it holds

$$
\mathbf{m}(A, \cdot) \leq \mathbf{m}(B, \cdot) \text { and } \mathbf{m}(\cdot, A) \geq \mathbf{m}(\cdot, B) .
$$

Now, let us consider $f: X \rightarrow[-1,1]$ and a bi-capacity $\mathbf{m}: Q(X) \rightarrow[-1,1]$. We denote $f\left(x_{i}\right)=f_{i}, i=1, \ldots, n$.

In the sequel, the class containing all functions $f: X \rightarrow[-1,1]$ will be denoted by $\mathcal{S}$. The class of all normalized bi-capacities $\mathbf{m}: Q(X) \rightarrow[-1,1]$, i.e. such that $\mathbf{m}(X, \emptyset)=1=-\mathbf{m}(\varnothing, X)$, will be denoted by $\mathcal{M}$. For each $A \in \mathcal{P}(X)$, the characteristic function is defined as usual by

$$
\chi_{A}(x)= \begin{cases}0, & x \notin A \\ 1, & x \in A .\end{cases}
$$

For each $(A, B) \in \mathcal{Q}(X), \chi_{(A, B)} \in \mathcal{S}$ is defined by

$$
\chi_{(A, B)}(x)=\left\{\begin{array}{rc}
1, & x \in A, \\
-1, & x \in B, \\
0, & x \notin A \cup B .
\end{array}\right.
$$

Obviously, for all $(A, B) \in \mathcal{Q}(X)$, it holds $\chi_{(A, B)}=\chi_{A}-\chi_{B}$.

For all $t \in] 0,1]$, the couple of sets $(\{x \in X \mid f(x) \geq t\},\{x \in X \mid f(x) \leq-t\})$, shortly denoted by $(\{f \geq t\},\{f \leq-t\})$ belongs to $Q(X)$. In order to ensure the same property for $t=0, \quad(\{f \geq 0\},\{f \leq 0\})$ will be interpreted as $(\{f \geq 0\}$, $\{f<0\})$.

The bipolar Shilkret integral was introduced in [12] and studied in [11].

Definition 2 For $f \in \mathcal{S}$ and $\mathbf{m} \in \mathcal{M}$, the bipolar Shilkret integral is defined by

$$
\operatorname{BSh}(f, \mathbf{m})=\mathbb{V}_{i=1}^{n}\left|f_{i}\right| \cdot \mathbf{m}\left(\left\{f \geq\left|f_{i}\right|\right\},\left\{f \leq-\left|f_{i}\right|\right\}\right) .
$$

Let $X_{n}$ be a finite set of cardinality $n$ for $n \in \mathbb{N}, \delta_{n}$ be the class of all functions from the set $X_{n}$ to the interval $[-1,1]$ and $\mathcal{M}_{n}$ be the class of all normalized bi-capacities on $\mathcal{Q}\left(X_{n}\right)$. The main properties of bipolar Shilkret integral $B C h: \cup_{n \in \mathbb{N}} \mathcal{S}_{n} \times \mathcal{M}_{n} \rightarrow[-1,1]$, given in [11], are:

(Sh1) $B S h\left(c \cdot \chi_{(A, B)}, \mathbf{m}\right)=c \cdot \mathbf{m}(A, B)$, for all $c \in[0,1]$ and for all $\mathbf{m} \in \mathcal{M}_{n}$, $(A, B) \in Q\left(X_{n}\right), n \in \mathbb{N}$

(Sh2) $B \operatorname{Sh}\left(f, \mathbf{m}_{1}\right) \geq B \operatorname{Sh}\left(g, \mathbf{m}_{2}\right)$, for all pairs $\left(f, \mathbf{m}_{1}\right) \in \mathcal{S}_{n_{1}} \times \mathcal{M}_{n_{1}}$, and $\left(g, \mathbf{m}_{2}\right) \in \mathcal{S}_{n_{2}} \times \mathcal{M}_{n_{2}}, n_{1}, n_{2} \in \mathbb{N}$ satisfying

$\mathbf{m}_{1}(\{f \geq t\},\{f \leq-t\}) \geq \mathbf{m}_{2}(\{g \geq t\},\{g \leq-t\})$,

for all $t \in] 0,1]$. 
The bipolar Sugeno integral was initially introduced in [12] and studied in [11].

Definition 3 For $f \in \mathcal{S}$ and $\mathbf{m} \in \mathcal{M}$, the bipolar Sugeno integral is given by

$$
\operatorname{BSu}(f, \mathbf{m})=\mathbb{V}_{i=1}^{n}\left|f_{i}\right| \mathbb{A} \mathbf{m}\left(\left\{f \geq\left|f_{i}\right|\right\},\left\{f \leq-\left|f_{i}\right|\right\}\right) .
$$

The bipolar Sugeno integral $B S u: \cup_{n \in \mathbb{N}} \mathcal{S}_{n} \times \mathcal{M}_{n} \rightarrow[-1,1]$ possesses the following properties:

(Su1) $B S u\left(c \cdot \chi_{(A, B)}, \mathbf{m}\right)=c \mathbb{A} \mathbf{m}(A, B)$, for all $c \in[0,1]$ and for all $\mathbf{m} \in \mathcal{M}_{n}$, $(A, B) \in Q\left(X_{n}\right), n \in \mathbb{N}$

(Su2) $B S u\left(f, \mathbf{m}_{1}\right) \geq B S u\left(g, \mathbf{m}_{2}\right)$, for all pairs $\left(f, \mathbf{m}_{1}\right) \in \mathcal{S}_{n_{1}} \times \mathcal{M}_{n_{1}}$, and $\left(g, \mathbf{m}_{2}\right) \in \mathcal{S}_{n_{2}} \times \mathcal{M}_{n_{2}}, n_{1}, n_{2} \in \mathbb{N}$ such that (1) is fulfilled for all $\left.\left.t \in\right] 0,1\right]$.

Finally, let us recall the definition of the bipolar Choquet integral. The bipolar Choquet integral was initially introduced in $[8,10]$ and studied in [11].

Definition 4 For $f \in \mathcal{S}$ and $\mathbf{m} \in \mathcal{M}$, the bipolar Choquet integral is defined by

$$
B C h(f, \mathbf{m})=\sum_{i=1}^{n}\left(\left|f_{\alpha(i)}\right|-\left|f_{\alpha(i-1)}\right|\right) \cdot \mathbf{m}\left(\left\{f \geq\left|f_{\alpha(i)}\right|\right\},\left\{f \leq-\left|f_{\alpha(i)}\right|\right\}\right),
$$

where a permutation of indexes $\alpha=(\alpha(1), \ldots \alpha(n))$ is related to non-decreasing order of values $\left|f\left(x_{i}\right)\right|=\left|f_{i}\right|$, i.e. such that $0 \leq\left|f_{\alpha(1)}\right| \leq\left|f_{\alpha(2)}\right| \leq \cdots \leq\left|f_{\alpha(n)}\right|$, $\left|f_{\alpha(0)}\right|=0$.

The bipolar Choquet integral $B C h: \cup_{n \in \mathbb{N}} \mathcal{S}_{n} \times \mathcal{M}_{n} \rightarrow[-1,1]$ has the next properties:

(Ch1) $B C h\left(c \cdot\left(\chi_{(A, B)}\right), \mathbf{m}\right)=c \cdot \mathbf{m}(A, B)$, for all $c \in[0,1]$ and for all $\mathbf{m} \in \mathcal{M}_{n}$, $(A, B) \in Q\left(X_{n}\right), n \in \mathbb{N}$;

(Ch2) $B C h\left(f, \mathbf{m}_{1}\right) \geq B C h\left(g, \mathbf{m}_{2}\right)$, for all pairs $\left(f, \mathbf{m}_{1}\right) \in \mathcal{S}_{n_{1}} \times \mathcal{M}_{n_{1}}$, and $\left(g, \mathbf{m}_{2}\right) \in S_{n_{2}} \times M_{n_{2}}, n_{1}, n_{2} \in \mathbb{N}$ such that (1) is fulfilled for all $\left.\left.t \in\right] 0,1\right]$.

By Theorem 2 from [11], the properties (Sh1), (Sh2), (Su1), (Su2), (Ch1) and (Ch2), ensure that each of bipolar integrals

$$
B S u, B S h, B C h: \cup_{n \in \mathbb{N}} \delta_{n} \times \mathcal{M}_{n} \rightarrow[-1,1]
$$

is the $[-1,1]$-valued bipolar universal integral introduced and studied in [11] (for more details we refer to [7, 8, 10-12]). 


\section{Jensen Inequality for the Bipolar Shilkret and Sugeno Integrals}

In this section, we present the results based on [29]. The Jensen type inequality for the bipolar Shilkret and Sugeno integrals is considered. Further, we will observe conditions under which the reversed Jensen type inequality for these integrals is valid.

We have the following lemma. We refer to [30] for details of its proof.

\section{Lemma 1}

(i) For any strictly increasing and odd function $\varphi, \varphi:[-1,1] \rightarrow[-1,1]$, it holds

$$
\varphi(|x|)=|\varphi(x)| .
$$

(ii) For any strictly increasing and odd function $\varphi, \varphi:[-1,1] \rightarrow[-1,1]$, and for any $f \in \mathcal{S}$, it holds:

$$
\left\{f \geq\left|f_{i}\right|\right\}=\left\{\varphi(f) \geq\left|\varphi\left(f_{i}\right)\right|\right\} \text { and }\left\{f \leq-\left|f_{i}\right|\right\}=\left\{\varphi(f) \leq-\left|\varphi\left(f_{i}\right)\right|\right\},
$$
for $i=1,2, \ldots, n$.

(iii) For any strictly increasing function $\varphi, \varphi:[-1,1] \rightarrow[-1,1]$, it holds

$$
\varphi\left(\bigwedge_{i=1}^{n} x_{i}\right)=\bigwedge_{i=1}^{n} \varphi\left(x_{i}\right) \text { and } \varphi\left(\bigvee_{i=1}^{n} x_{i}\right)=\bigvee_{i=1}^{n} \varphi\left(x_{i}\right)
$$

for all $x_{i} \in[0,1], i=1, \ldots, n$.

(iv) For any function $\varphi, \varphi:[-1,1] \rightarrow[-1,1]$, such that $\varphi$ is a convex function on $[0,1]$ and concave on $[-1,0]$, and $\varphi(0)=0$, it holds

$$
\varphi(\lambda \cdot x) \leq \lambda \cdot \varphi(x)
$$

for all $x \in[0,1]$ and $\lambda \in[0,1]$, and

$$
\varphi(\lambda \cdot x) \geq \lambda \cdot \varphi(x)
$$

for all $x \in[-1,0]$ and $\lambda \in[0,1]$.

In the following theorem, we consider the Jensen type inequality related to the bipolar Shilkret integral.

Theorem 1 Let $\varphi:[-1,1] \rightarrow[-1,1]$ be an odd and strictly increasing function, which is convex on $[0,1]$. For all $f \in \mathcal{S}$ and $\mathbf{m} \in \mathcal{M}$, if $B \operatorname{Sh}(\varphi(f), \mathbf{m}) \neq 0$, it holds

$$
\varphi(|B S h(f, \mathbf{m})|) \leq|B S h(\varphi(f), \mathbf{m})| .
$$

Proof. The bipolar Shilkret integral can be rewritten as

$$
B S h(f, \mathbf{m})=(\mathcal{S B S h}(f)) \underset{i=1}{n}\left|f_{i}\right| \cdot\left|\mathbf{m}\left(\left\{f \geq\left|f_{i}\right|\right\},\left\{f \leq-\left|f_{i}\right|\right\}\right)\right|,
$$


where, the notation $S B S h_{\mathbf{m}}(f)$ is used to denote the signum of the bipolar Shilkret integral of $f$ w.r.t. $\mathbf{m}$.

Let us assume that it holds $S B S h_{\mathbf{m}}(\varphi(f)) \neq 0$. Due to (3) and Lemma 1 (iii), we obtain

$$
\begin{aligned}
\varphi(|B S h(f, \mathbf{m})|) & =\varphi\left(\bigvee_{i=1}^{n}\left|f_{i}\right| \cdot\left|\mathbf{m}\left(\left\{f \geq\left|f_{i}\right|\right\},\left\{f \leq-\left|f_{i}\right|\right\}\right)\right|\right) \\
& =\bigvee_{i=1}^{n} \varphi\left(\left|f_{i}\right| \cdot\left|\mathbf{m}\left(\left\{f \geq\left|f_{i}\right|\right\},\left\{f \leq-\left|f_{i}\right|\right\}\right)\right|\right) .
\end{aligned}
$$

Further, according to Lemma 1 (iv), (ii) and (i) we have

$$
\begin{aligned}
\varphi(|B S h(f, \mathbf{m})|) & \leq \mathrm{V}_{i=1}^{n} \varphi\left(\left|f_{i}\right|\right) \cdot\left|\mathbf{m}\left(\left\{\varphi(f) \geq\left|\varphi\left(f_{i}\right)\right|\right\},\left\{\varphi(f) \leq-\left|\varphi\left(f_{i}\right)\right|\right\}\right)\right| \\
& =\bigvee_{i=1}^{n}\left|\varphi\left(f_{i}\right)\right| \cdot\left|\mathbf{m}\left(\left\{\varphi(f) \geq\left|\varphi\left(f_{i}\right)\right|\right\},\left\{\varphi(f) \leq-\left|\varphi\left(f_{i}\right)\right|\right\}\right)\right| \\
& =|B S h(\varphi(f), \mathbf{m})| .
\end{aligned}
$$

Corollary 1 Let $\varphi:[-1,1] \rightarrow[-1,1], \varphi(1)=1$, be an odd and strictly increasing function, which is concave on $[0,1]$. For all $f \in \mathcal{S}$ and $\mathbf{m} \in \mathcal{M}$, if $\operatorname{BSh}(f, \mathbf{m}) \neq 0$, it holds

$$
\varphi(|B S h(f, \mathbf{m})|) \geq|B S h(\varphi(f), \mathbf{m})| .
$$

Proof. Using the fact that $\varphi^{-1}$ is odd and strictly increasing on $[-1,1]$ and convex on $[0,1]$, we obtain

$$
\varphi^{-1}(|B S h(\varphi(f), \mathbf{m})|) \leq\left|B S h\left(\varphi^{-1}(\varphi(f)), \mathbf{m}\right)\right|=|B S h(f, \mathbf{m})|,
$$

thus the claim is valid.

Notice that the stated inequality holds even if the condition $\varphi(1)=1$ is omitted.

Example 1 Let $X=\left\{x_{1}, x_{2}, x_{3}\right\}, \mathbf{m} \in \mathcal{M}$ such that $\mathbf{m}\left(\varnothing,\left\{x_{3}\right\}\right)=-0.5$, $\mathbf{m}\left(\left\{x_{2}\right\},\left\{x_{3}\right\}\right)=0.4$ and $\mathbf{m}\left(\left\{x_{1}, x_{2}\right\},\left\{x_{3}\right\}\right)=0.6$. Let $f\left(x_{1}\right)=0, f\left(x_{2}\right)=0.6$, $f\left(x_{3}\right)=-0.8$. By Definition 3, we get:

$$
\begin{aligned}
B S h(f, \mathbf{m}) & =\left(0 \cdot \mathbf{m}\left(\left\{x_{1}, x_{2}\right\},\left\{x_{3}\right\}\right)\right) \mathbb{V}\left(0.6 \cdot \mathbf{m}\left(\left\{x_{2}\right\},\left\{x_{3}\right\}\right)\right) \mathbb{V}\left(0.8 \cdot \mathbf{m}\left(\varnothing,\left\{x_{3}\right\}\right)\right) \\
& =(0 \cdot 0.6) \mathbb{V}(0.6 \cdot 0.4) \mathbb{V}(0.8 \cdot(-0.5)) \\
& =0 \mathbb{V} 0.24 \mathbb{V}(-0.4)=-0.4 .
\end{aligned}
$$

(i) Let $\varphi(x)=\frac{x}{2-|x|}, x \in[-1,1]$. Obviously, $\varphi$ is convex on $[0,1]$.

$$
\varphi(B S h(f, \mathbf{m}))=\frac{-0.4}{2-0.4}=-0.25 \text {. }
$$

Now, compute the related bipolar Shilkret integral of $\varphi(f)$ :

$$
\begin{aligned}
B S h(\varphi(f), \mathbf{m}) & =\left(\varphi(0) \cdot \mathbf{m}\left(\left\{x_{1}, x_{2}\right\},\left\{x_{3}\right\}\right)\right) \mathbb{V}\left(\varphi(0.6) \cdot \mathbf{m}\left(\left\{x_{2}\right\},\left\{x_{3}\right\}\right)\right) \\
& \mathbb{V}\left(\varphi(0.8) \cdot \mathbf{m}\left(\varnothing,\left\{x_{3}\right\}\right)\right) \\
& =(\varphi(0.6) \cdot 0.4) \mathbb{V}(\varphi(0.8) \cdot(-0.5)) \\
& \approx 0.17143 \mathbb{V}(-0.33333)=-0.33333 .
\end{aligned}
$$


We have $\varphi(|B S h(f, \mathbf{m})|)=\frac{1}{4}<\frac{1}{3}=|B \operatorname{Sh}(\varphi(f), \mathbf{m})|$, i.e. in this case the inequality (2) is strict.

(ii) Let $\varphi(x)=k x, x \in[-1,1]$, where $0<k \leq 1$. We obtain

$$
\varphi(B \operatorname{Sh}(f, \mathbf{m}))=-0.4 k \text {. }
$$

On the other hand, the bipolar Shilkret integral of a function $\varphi(f)$ is:

$$
\begin{aligned}
B S h(\varphi(f), \mathbf{m}) & =(0 \cdot 0.6) \mathbb{V}(0.6 k \cdot 0.4) \mathbb{V}(0.8 k \cdot(-0.5)) \\
& =0.24 k \mathbb{V}(-0.4 k) \\
& =-0.4 k .
\end{aligned}
$$

In this case, we have the equality $\varphi(|B \operatorname{Sh}(f, \mathbf{m})|)=|\operatorname{BSh}(\varphi(f), \mathbf{m})|=0.4 k$.

(iii) Let $\varphi(x)=\frac{2 x}{1+|x|}, x \in[-1,1]$. Obviously, $\varphi$ is concave on $[0,1]$. We obtain

$$
\varphi(B \operatorname{Sh}(f, \mathbf{m}))=\frac{2 \cdot(-0.4)}{1+|-0.4|}=-\frac{4}{7} \approx-0.57143 .
$$

Now, compute the related bipolar Shilkret integral of $\varphi(f)$ :

$$
\begin{aligned}
B S h(\varphi(f), \mathbf{m}) & =\left(\varphi(0) \cdot \mathbf{m}\left(\left\{x_{1}, x_{2}\right\},\left\{x_{3}\right\}\right)\right) \mathbb{V}\left(\varphi(0.6) \cdot \mathbf{m}\left(\left\{x_{2}\right\},\left\{x_{3}\right\}\right)\right) \\
& \mathbb{V}\left(\varphi(0.8) \cdot \mathbf{m}\left(\varnothing,\left\{x_{3}\right\}\right)\right) \\
& =(\varphi(0.6) \cdot 0.4) \mathbb{V}(\varphi(0.8) \cdot(-0.5)) \\
& \approx 0.3 \mathbb{V}(-0.44444)=-0.44444 .
\end{aligned}
$$

Hence, $\varphi(|B S h(f, \mathbf{m})|)=\frac{4}{7}>\frac{4}{9}=|B S h(\varphi(f), \mathbf{m})|$, i.e. the inequality given in Corollary 2 is strict.

Notice that under the conditions given in Theorem 1, if $\mathcal{S} B S h_{\mathbf{m}}(\varphi(f))=0$, then the inequality (2) need not be satisfied, that is illustrated in the following example.

Example 2 Consider $X=\left\{x_{1}, x_{2}, x_{3}\right\}$ and the function $f$ given by $f\left(x_{1}\right)=\sqrt[5]{0.3}$, $f\left(x_{2}\right)=-\sqrt[5]{0.6}, f\left(x_{3}\right)=\sqrt[5]{0.1}$, and $\varphi(x)=x^{5}, x \in[-1,1]$.

(i) Let $\mathbf{m} \in \mathcal{M}$, such that $\mathbf{m}\left(\left\{x_{1}, x_{3}\right\},\left\{x_{2}\right\}\right)=1, \mathbf{m}\left(\left\{x_{1}\right\},\left\{x_{2}\right\}\right)=0.8$ and $\mathbf{m}\left(\varnothing,\left\{x_{2}\right\}\right)=-0.4$. We have

$$
\begin{aligned}
B S h(f, \mathbf{m}) & =\left(\sqrt[5]{0.3} \cdot \mathbf{m}\left(\left\{x_{1}\right\},\left\{x_{2}\right\}\right)\right) \mathbb{V}\left(\sqrt[5]{0.6} \cdot \mathbf{m}\left(\varnothing,\left\{x_{2}\right\}\right)\right) \\
& \mathbb{V}\left(\sqrt[5]{0.1} \cdot \mathbf{m}\left(\left\{x_{1}, x_{3}\right\},\left\{x_{2}\right\}\right)\right) \\
& =(\sqrt[5]{0.3} \cdot 0.8) \mathbb{V}(\sqrt[5]{0.6} \cdot(-0.4))(\sqrt[5]{0.1} \cdot 1) \\
& \approx 0.62880 \mathbb{V}(-0.36115) \mathbb{V} 0.63096 \\
& =0.63096
\end{aligned}
$$


thus, $\varphi(B \operatorname{Sh}(f, \mathbf{m}))=0.1$ and

$$
\begin{aligned}
B S h(\varphi(f), \mathbf{m}) & \left.=\left(0.3 \cdot \mathbf{m}\left(\left\{x_{1}\right\},\left\{x_{2}\right\}\right)\right) \mathbb{V}\left(0.6 \cdot \mathbf{m}\left(\varnothing,\left\{x_{2}\right\}\right\}\right)\right) \\
& \mathbb{V}\left(0.1 \cdot \mathbf{m}\left(\left\{x_{1}, x_{3}\right\},\left\{x_{2}\right\}\right)\right) \\
& =(0.3 \cdot 0.8) \mathbb{V}(0.6 \cdot(-0.4)) \mathbb{V}(0.1 \cdot 1) \\
& =0.24 \mathbb{V}(-0.24) \mathbb{V} 0.1 \\
& =0 .
\end{aligned}
$$

Hence, we have $\varphi(|B S h(f, \mathbf{m})|)>|B S h(\varphi(f), \mathbf{m})|=0$.

(ii) Let $\mathbf{m} \in \mathcal{M}$, such that $\mathbf{m}\left(\left\{x_{2}\right\},\left\{x_{1}, x_{3}\right\}\right)=-1, \mathbf{m}\left(\left\{x_{2}\right\},\left\{x_{1}\right\}\right)=-0.8$, $\mathbf{m}\left(\left\{x_{2}\right\}, \emptyset\right)=0.4$, and $g=-f$. We obtain:

$$
\begin{aligned}
\operatorname{BSh}(g, \mathbf{m}) & =\left(\sqrt[5]{0.3} \cdot \mathbf{m}\left(\left\{x_{2}\right\},\left\{x_{1}\right\}\right)\right) \mathbb{V}\left(\sqrt[5]{0.6} \cdot \mathbf{m}\left(\left\{x_{2}\right\}, \emptyset\right)\right) \\
& \mathbb{V}\left(\sqrt[5]{0.1} \cdot \mathbf{m}\left(\left\{x_{2}\right\},\left\{x_{1}, x_{3}\right\}\right)\right) \\
& =(\sqrt[5]{0.3} \cdot(-0.8)) \mathbb{V}(\sqrt[5]{0.6} \cdot 0.4) \mathbb{V}(\sqrt[5]{0.1} \cdot(-1)) \\
& \approx(-0.62880) \mathbb{V} 0.36115 \mathbb{V}(-0.63096)=-0.63096,
\end{aligned}
$$

therefore, $\varphi(B \operatorname{Sh}(g, \mathbf{m}))=-0.1$ and

$$
\begin{aligned}
\operatorname{BSh}(\varphi(g), \mathbf{m}) & =\left(0.3 \cdot \mathbf{m}\left(\left\{x_{2}\right\},\left\{x_{1}\right\}\right)\right) \mathbb{V}\left(0.6 \cdot \mathbf{m}\left(\left\{x_{2}\right\}, \emptyset\right)\right) \\
& \mathbb{V}\left(0.1 \cdot \mathbf{m}\left(\left\{x_{2}\right\},\left\{x_{1}, x_{3}\right\}\right)\right) \\
& =(0.3 \cdot(-0.8)) \mathbb{V}(0.6 \cdot 0.4)) \mathbb{V}(0.1 \cdot(-1)) \\
& =(-0.24) \mathbb{V} 0.24 \mathbb{V}(-0.1)=0 .
\end{aligned}
$$

Hence, we have $\varphi(|B \operatorname{Sh}(g, \mathbf{m})|)>|B \operatorname{Sh}(\varphi(g), \mathbf{m})|=0$.

(iii) If we take $\varphi(x)=x, \quad x \in[-1,1]$, same $\mathbf{m} \in \mathcal{M}$ as that given in (i), and $h \in \mathcal{M}$, given by $h\left(x_{1}\right)=0.3, h\left(x_{2}\right)=-0.6, h\left(x_{3}\right)=0.1$, then we get $\varphi(|B \operatorname{Sh}(h, \mathbf{m})|)=|B \operatorname{Sh}(\varphi(h), \mathbf{m})|=0$.

Now, we will observe conditions under which the Jensen type inequality for the bipolar Sugeno integral is valid.

Theorem 2 Let $\varphi:[-1,1] \rightarrow[-1,1]$ be an odd and strictly increasing function, such that $\varphi(x) \leq x$ on $[0,1]$. For all $f \in \mathcal{S}$ and $\mathbf{m} \in \mathcal{M}$, if $B S u(\varphi(f), \mathbf{m}) \neq 0$, then

$$
\varphi(|B S u(f, \mathbf{m})|) \leq|B S u(\varphi(f), \mathbf{m})| .
$$

Proof. The bipolar Sugeno integral can be expressed by:

$$
B S u(f, \mathbf{m})=\left(\mathcal{S B S h} h_{\mathbf{m}}(f)\right) \stackrel{\vee}{n}_{i=1}^{n}\left|f_{i}\right| \wedge\left|\mathbf{m}\left(\left\{f \geq\left|f_{i}\right|\right\},\left\{f \leq-\left|f_{i}\right|\right\}\right)\right|,
$$


where, the notation $S B S u_{\mathbf{m}}(f)$ is used to denote the signum of the bipolar Sugeno integral of $f$ w.r.t. $\mathbf{m}$.

Let $\mathcal{S} B S u_{\mathbf{m}}(\varphi(f)) \neq 0$. By (4) and Lemma 1 (iii) we get

$$
\begin{aligned}
\varphi(|B S u(f, \mathbf{m})|) & =\varphi\left(\bigvee_{i=1}^{n}\left|f_{i}\right| \wedge\left|\mathbf{m}\left(\left\{f \geq\left|f_{i}\right|\right\},\left\{f \leq-\left|f_{i}\right|\right\}\right)\right|\right) \\
& =\bigvee_{i=1}^{n} \varphi\left(\left|f_{i}\right| \wedge\left|\mathbf{m}\left(\left\{f \geq\left|f_{i}\right|\right\},\left\{f \leq-\left|f_{i}\right|\right\}\right)\right|\right) \\
& =\bigvee_{i=1}^{n} \varphi\left(\left|f_{i}\right|\right) \wedge \varphi\left(\left|\mathbf{m}\left(\left\{f \geq\left|f_{i}\right|\right\},\left\{f \leq-\left|f_{i}\right|\right\}\right)\right|\right) .
\end{aligned}
$$

Further, due to the condition that $\varphi(x) \leq x$ on $[0,1]$ and Lemma 1 (ii), we have

$$
\begin{aligned}
\varphi(|B S u(f, \mathbf{m})|) & \leq \bigvee_{i=1}^{n} \varphi\left(\left|f_{i}\right|\right) \wedge\left|\mathbf{m}\left(\left\{f \geq\left|f_{i}\right|\right\},\left\{f \leq-\left|f_{i}\right|\right\}\right)\right| \\
& =\bigvee_{i=1}^{n}\left|\varphi\left(f_{i}\right)\right| \wedge\left|\mathbf{m}\left(\left\{\varphi(f) \geq\left|\varphi\left(f_{i}\right)\right|\right\},\left\{\varphi(f) \leq-\left|\varphi\left(f_{i}\right)\right|\right\}\right)\right| \\
& =|B S u(\varphi(f), \mathbf{m})| .
\end{aligned}
$$

With a similar consideration as in the proof of Corollary 1, we have the next consequence of Theorem 2 .

Corollary 2 Let $\varphi:[-1,1] \rightarrow[-1,1], \varphi(1)=1$, be an odd and strictly increasing function, such that $\varphi(x) \geq x$ on $[0,1]$, which is concave on $[0,1]$. For all $f \in \mathcal{S}$ and $\mathbf{m} \in \mathcal{M}$, if $B S u(f, \mathbf{m}) \neq 0$, it holds

$$
\varphi(|B S u(f, \mathbf{m})|) \geq|B S u(\varphi(f), \mathbf{m})| .
$$

Similarly as in the case of the bipolar Shilkret integral, the inequality holds even if the condition $\varphi(1)=1$ is omitted.

Example 3 Let us consider $f$ and $\mathbf{m}$ from Example 1.

(i) Let $\varphi(x)=\operatorname{sign}(x) \ln (1+|x|), x \in[-1,1]$. Obviously, $\varphi(x) \leq x$, for each $x \in[0,1]$. We have

$$
\begin{aligned}
& B S u(f, \mathbf{m})=\left(0 \mathbb{A} \mathbf{m}\left(\left\{x_{1}, x_{2}\right\},\left\{x_{3}\right\}\right)\right) \mathbb{V}\left(0.6 \mathbb{A} \mathbf{m}\left(\left\{x_{2}\right\},\left\{x_{3}\right\}\right)\right) \\
& \mathbb{V}\left(0.8 \wedge \mathbf{m}\left(\varnothing,\left\{x_{3}\right\}\right)\right) \\
& =(0 \wedge 0.6) \mathbb{V}(0.6 \AA 0.4) \mathbb{V}(0.8 \wedge(-0.5)) \\
& =0 \quad 0.4 \mathbb{V}(-0.5)=-0.5 \text {, }
\end{aligned}
$$

and therefore $\varphi(B S u(f, \mathbf{m}))=-\ln (1+0.5) \approx-0.40547$. On the other hand, we have

$$
\begin{aligned}
B S u(\varphi(f), \mathbf{m}) & =(\varphi(0) \mathbb{\wedge} 0.6) \mathbb{V}(\varphi(0.6) \mathbb{\wedge} 0.4) \mathbb{V}(\varphi(0.8) \mathbb{\wedge}(-0.5)) \\
& =(0.47 \mathbb{\wedge} 0.4) \mathbb{V}(0.58779 \mathbb{\wedge}(-0.5)) \\
& =0.4 \mathbb{V}(-0.5)=-0.5 .
\end{aligned}
$$


Hence,

$$
\varphi(|B S u(f, \mathbf{m})|)=0.40547 \leq 0.5=|B S u(\varphi(f), \mathbf{m})|,
$$

i.e. the inequality stated in Theorem 2 is fulfilled.

(ii) Let $\varphi(x)=\frac{2 x}{1+|x|}, x \in[-1,1]$. Obviously, $\varphi(x) \geq x$, for each $x \in[0,1]$.

We have $\varphi(B S u(f, \mathbf{m}))=\frac{2 \cdot(-0.5)}{1+|-0.5|}=-\frac{2}{3}$ and

$$
\begin{aligned}
B S u(\varphi(f), \mathbf{m}) & =(\varphi(0) \mathbb{\wedge} 0.6) \mathbb{V}(\varphi(0.6) \wedge 0.4) \mathbb{V}(\varphi(0.8) \wedge(-0.5)) \\
& \approx(0.75 \mathbb{\wedge} 0.4) \mathbb{V}(0.88889 \mathbb{\wedge}(-0.5)) \\
& =0.4 \mathbb{V}(-0.5)=-0.5
\end{aligned}
$$

Hence,

$$
\varphi(|B S u(f, \mathbf{m})|)=\frac{2}{3} \geq \frac{1}{2}=|B S u(\varphi(f), \mathbf{m})|,
$$

i.e. the inequality stated in Corollary 2 is fulfilled.

Similarly as in Example 2 with the bipolar Shilkret integral, under the conditions of Theorem 2, if $\mathcal{S} B S u_{\mathbf{m}}(\varphi(f))=0$, the reversed inequality holds (see Example 10 in [29]):

$$
\varphi(|B S u(f, \mathbf{m})|) \geq|B S u(\varphi(f), \mathbf{m})|=0 .
$$

\section{Jensen Inequality for the Bipolar Choquet Integral}

The bipolar Choquet integral is the most prominent member of the class of bipolar Choquet g-integrals introduced in [17]. Therefore, based on [17], in this section, the Jensen type inequality for the bipolar Choquet integral is considered. Here, we will give the complete proof of the main theorem, and present the conditions under which the reversed Jensen type inequality for this integral is valid.

For $f \in \mathcal{S}$, denote

$$
\begin{gathered}
X^{+}=\left\{x_{i} \in X \mid f\left(x_{i}\right)>0\right\}, \quad X^{-}=\left\{x_{i} \in X \mid f\left(x_{i}\right)<0\right\}, \\
X^{0}=\left\{x_{i} \in X \mid f\left(x_{i}\right)=0\right\}, \operatorname{supp}(f)=X^{+} \cup X^{-}=X \backslash X^{0}, \\
X^{+0}=X^{+} \cup X^{0} \text { and } X^{-0}=X^{-} \cup X^{0} .
\end{gathered}
$$

Let $\mathbf{m} \in \mathcal{M}$ and $f \in \mathcal{S}$. Define set functions $\mu_{f^{+}}, \tilde{\mu}_{f^{+}}: \mathcal{P}(X) \rightarrow[-1,1]$ by

$$
\begin{aligned}
\mu_{f^{+}}(A) & =\mathbf{m}\left(A \cap X^{+0}, A \cap X^{-}\right) \\
\tilde{\mu}_{f^{+}}(A) & =\mathbf{m}\left(A \cap X^{+}, A \cap X^{-0}\right) .
\end{aligned}
$$


Let $\widehat{\mathbf{m}}(A, B)=-\mathbf{m}(B, A)$, for all $(A, B) \in Q(X)$. First, by Definition 2 it can be easily verified that $\widehat{\mathbf{m}} \in \mathcal{M}$. Further, for all $A \in \mathcal{P}(X)$ we have:

$$
\begin{aligned}
& \mu_{f^{+}}(A)=-\hat{\mu}_{(-f)^{+}}(A), \text { if } A \subset \operatorname{supp}(f) ; \\
& \mu_{f^{+}}(A)=-\tilde{\hat{\mu}}_{(-f)^{+}}(A) .
\end{aligned}
$$

Obviously, any function $f \in \mathcal{S}$ can be expressed by:

$$
\begin{aligned}
f & =\sum_{i=1}^{n}\left(\left|f_{\alpha(i)}\right|-\left|f_{\alpha(i-1)}\right|\right) \cdot \chi_{\left(A_{i} \cap X^{+0}, A_{i} \cap X^{-}\right)} \\
& =\sum_{i=1}^{n}\left(\left|f_{\alpha(i)}\right|-\left|f_{\alpha(i-1)}\right|\right) \cdot \chi_{\left(A_{i} \cap X^{+}, A_{i} \cap X^{-0}\right)},
\end{aligned}
$$

where $\left|f_{\alpha(i)}\right| \leq\left|f_{\alpha(j)}\right|$, for all $i \leq j, \quad f_{\alpha(0)}=0$, and $A_{i}=\left\{x_{\alpha(i)}, \ldots, x_{\alpha(n)}\right\}$, $i=1, \ldots, n$.

Now, for computing the bipolar Choquet integral we can use the formula from the next proposition.

Proposition 1 Let $\varphi:[-1,1] \rightarrow[-1,1]$ be odd and strictly increasing function, $f \in \mathcal{S}, \varphi(f)=\varphi \circ f \in \mathcal{S}$ and $\mathbf{m} \in \mathcal{M}$. Then:

$$
\begin{aligned}
B C h(\varphi(f), \mathbf{m}) & =\sum_{i=1}^{n}\left(\varphi\left(\left|f_{\alpha(i)}\right|\right)-\varphi\left(\left|f_{\alpha(i-1)}\right|\right)\right) \cdot \mu_{f^{+}}\left(A_{i}\right) \\
& =\sum_{i=1}^{n}\left(\varphi\left(\left|f_{\alpha(i)}\right|\right)-\varphi\left(\left|f_{\alpha(i-1)}\right|\right)\right) \cdot \tilde{\mu}_{f^{+}}\left(A_{i}\right) \\
& =\sum_{i=1}^{n} \varphi\left(\left|f_{\alpha(i)}\right|\right) \cdot\left(\mu_{f^{+}}\left(A_{i}\right)-\mu_{f^{+}}\left(A_{i+1}\right)\right),
\end{aligned}
$$

for any permutation $\alpha$ of indexes such that $\left|f_{\alpha(i)}\right| \leq\left|f_{\alpha(j)}\right|$, for all $i \leq j$, $f_{\alpha(0)}=0, A_{i}=\left\{x_{\alpha(i)}, \ldots, x_{\alpha(n)}\right\}, i=1, \ldots, n$, and $A_{n+1}=\emptyset$.

Proof. Let $f \in \mathcal{S}$ and $\mathbf{m} \in \mathcal{M}$. Let $\varphi$ be odd and strictly increasing and $\varphi(f)=$ $\varphi \circ f \in \mathcal{S}$. For an arbitrary permutation of indexes $\alpha$, such that $\left|f_{\alpha(i)}\right| \leq\left|f_{\alpha(j)}\right|$, for all $i \leq j, f_{\alpha(0)}=0$, let $K=\left\{j|| f_{\alpha(j)}|\neq| f_{\alpha(j-1)} \mid\right\}$. For all $i \in K$, we get:

$$
\mathbf{m}\left(\left\{f \geq\left|f_{\alpha(i)}\right|\right\},\left\{f \leq-\left|f_{\alpha(i)}\right|\right\}\right)=\mu_{f^{+}}\left(A_{i}\right)=\tilde{\mu}_{f^{+}}\left(A_{i}\right) .
$$

By Lema 1, we have $\varphi(0)=0, \varphi(|x|)=|\varphi(x)|$, for all $x \in[-1,1]$, and $\mu_{f^{+}}=$ $\mu_{(\varphi \circ f)}+$. Therefore

$$
\begin{aligned}
B C h(\varphi(f), \mathbf{m}) & =\sum_{i \in K}\left(\left|\varphi\left(f_{\alpha(i)}\right)\right|-\left|\varphi\left(f_{\alpha(i-1)}\right)\right|\right) \cdot \mu_{(\varphi \circ f)^{+}}\left(A_{i}\right) \\
& =\sum_{i \in K}\left(\varphi\left(\left|f_{\alpha(i)}\right|\right)-\varphi\left(\left|f_{\alpha(i-1)}\right|\right)\right) \cdot \mu_{f^{+}}\left(A_{i}\right) \\
& =\sum_{i=1}^{n}\left(\varphi\left(\left|f_{\alpha(i)}\right|\right)-\varphi\left(\left|f_{\alpha(i-1)}\right|\right)\right) \cdot \mu_{f^{+}}\left(A_{i}\right) \\
& =\sum_{i=1}^{n} \varphi\left(\left|f_{\alpha(i)}\right|\right) \cdot\left(\mu_{f^{+}}\left(A_{i}\right)-\mu_{f^{+}}\left(A_{i+1}\right)\right),
\end{aligned}
$$

where $A_{i}=\left\{x_{\alpha(i)}, \ldots, x_{\alpha(n)}\right\}$, for $i \geq 2, A_{1}=X, A_{n+1}=\emptyset$. Hence, (9) and (11) are valid. Due to $\mu_{f^{+}}(A)=\tilde{\mu}_{f^{+}}(A)$, for all $A \subset \operatorname{supp}(f)$, we obtain (10). 
Example 4 ([17]) Consider $X=\left\{x_{1}, x_{2}, x_{3}, x_{4}\right\}$ and $f: X \rightarrow[-1,1]$ such that $f\left(x_{1}\right)=0.2, f\left(x_{2}\right)=-0.2, \quad f\left(x_{3}\right)=0, f\left(x_{4}\right)=-0.4$. Obviously, we get $X^{+0}=\left\{x_{1}, x_{3}\right\}, X^{-}=\left\{x_{2}, x_{4}\right\}$. Let $\mathbf{m} \in \mathcal{M}, \operatorname{card}(Q(X))=3^{4}=81$.

Consider $\varphi(x)=x^{k}, x \in \mathbb{R}$, for odd $k \in \mathbb{N}$. For $\alpha_{1}=(3,1,2,4)$, we have $\left|\varphi\left(f_{3}\right)\right| \leq\left|\varphi\left(f_{1}\right)\right| \leq\left|\varphi\left(f_{2}\right)\right| \leq\left|\varphi\left(f_{4}\right)\right|$. Using Proposition 1, we get:

$$
\begin{aligned}
& B C h(\varphi(f), \mathbf{m})= \\
& \quad=(|0|-|0|) \cdot \mu_{f^{+}}\left(\left\{x_{3}, x_{1}, x_{2}, x_{4}\right\}\right)+\left(|0.2|^{k}-|0|\right) \cdot \mu_{f^{+}}\left(\left\{x_{1}, x_{2}, x_{4}\right\}\right)+ \\
& +\left(|-0.2|^{k}-|0.2|^{k}\right) \cdot \mu_{f^{+}}\left(\left\{x_{2}, x_{4}\right\}\right)+\left(|-0.4|^{k}-|-0.2|^{k}\right) \cdot \mu_{f^{+}}\left(\left\{x_{4}\right\}\right) \\
& \quad=|0.2|^{k} \cdot \mu_{f^{+}}\left(\left\{x_{1}, x_{2}, x_{4}\right\}\right)+\left(|-0.4|^{k}-|-0.2|^{k}\right) \cdot \mu_{f^{+}}\left(\left\{x_{4}\right\}\right) .
\end{aligned}
$$

For $\alpha_{2}=(3,2,1,4), \alpha_{1} \neq \alpha_{2}$, it holds $\left|\varphi\left(f_{3}\right)\right| \leq\left|\varphi\left(f_{2}\right)\right| \leq\left|\varphi\left(f_{1}\right)\right| \leq\left|\varphi\left(f_{4}\right)\right|$, and by Proposition 1, we obtain the same result:

$$
\begin{aligned}
& B C h(\varphi(f), \mathbf{m})= \\
& \quad=\left(|-0.2|^{k}-|0|\right) \cdot \mu_{f^{+}}\left(\left\{x_{2}, x_{1}, x_{4}\right\}\right)+\left(|-0.4|^{k}-|0.2|^{k}\right) \cdot \mu_{f^{+}}\left(\left\{x_{4}\right\}\right) \\
& \quad=|-0.2|^{k} \cdot \mu_{f^{+}}\left(\left\{x_{2}, x_{1}, x_{4}\right\}\right)+\left(|-0.4|^{k}-|0.2|^{k}\right) \cdot \mu_{f^{+}}\left(\left\{x_{4}\right\}\right) .
\end{aligned}
$$

Notice that $\mu_{f^{+}}\left(\left\{x_{2}, x_{1}, x_{4}\right\}\right)=\mu_{f^{+}}\left(\left\{x_{1}, x_{2}, x_{4}\right\}\right)=\mathbf{m}\left(\left\{x_{1}\right\},\left\{x_{2}, x_{4}\right\}\right) \quad$ and $\mu_{f^{+}}\left(\left\{x_{4}\right\}\right)=\mathbf{m}\left(\varnothing,\left\{x_{4}\right\}\right)$.

It should be noticed that by Proposition 1 , for $\varphi(x)=x, x \in[-1,1]$, for $f \in \mathcal{S}$, m $\in \mathcal{M}$, with $\alpha$ and $A_{i}$ previously described, we have:

$$
B C h(f, \mathbf{m})=\sum_{i=1}^{n}\left|f_{\alpha(i)}\right| \cdot\left(\mu_{f^{+}}\left(A_{i}\right)-\mu_{f^{+}}\left(A_{i+1}\right)\right) .
$$

Now, the proof of the next claim easily follows from (12) and (7).

Proposition 2 ([17]) Let $f \in \mathcal{S}$ and $\mathbf{m} \in \mathcal{M}$. Then

$$
B C h(-f, \mathbf{m})=-B C h(f, \widehat{\mathbf{m}}),
$$

where $\widehat{\mathbf{m}} \in \mathcal{M}$ is given by $\widehat{\mathbf{m}}(A, B)=-\mathbf{m}(B, A)$, for all $(A, B) \in Q(X)$.

Jensen type inequality for the bipolar Choquet integral is presented in the next theorem. Here, we give its complete proof. Very recently, the similar proving technic has been presented in [16], however, the following result is more general in the sense that the underlining set functions $\mu_{f^{+}}$and $\tilde{\mu}_{f^{+}}$given by (5) and (6) associated to a bi-capacity need not to be the difference of two fuzzy measures.

Theorem 3 Let $\varphi:[-1,1] \rightarrow[-1,1]$ be an odd and strictly increasing function, such that it is convex on $[0,1]$, and $c>0$. Let $f \in \mathcal{S}$ and $\mathbf{m} \in \mathcal{M}$. If there exists a permutation $\alpha$ of indexes such that $\left|f_{\alpha(i)}\right| \leq\left|f_{\alpha(j)}\right|$, for all $i \leq j$, and for all $i, j=1, \ldots, n$ it holds $\mu_{f^{+}}\left(A_{i}\right) \mu_{f^{+}}\left(A_{j}\right) \geq 0,\left|\mu_{f^{+}}\left(A_{i}\right)\right| \leq c$ and $\left|\mu_{f^{+}}\left(A_{1}\right)\right|=c$ or $\tilde{\mu}_{f^{+}}\left(A_{i}\right) \tilde{\mu}_{f^{+}}\left(A_{j}\right) \geq 0, \quad\left|\tilde{\mu}_{f^{+}}\left(A_{i}\right)\right| \leq c \quad$ and $\quad\left|\tilde{\mu}_{f^{+}}\left(A_{1}\right)\right|=c$, where $A_{i}=\left\{x_{\alpha(i)}, \ldots, x_{\alpha(n)}\right\}$, then 


$$
\varphi\left(\frac{1}{c} \cdot|B C h(f, \mathbf{m})|\right) \leq \frac{1}{c} \cdot|B C h(\varphi(f), \mathbf{m})| .
$$

Proof. Let $f \in \mathcal{S}$ and $\mathbf{m} \in \mathcal{M}$. Let $\alpha$ be desired permutation. We have two possible cases:

Case I For all $i=1, \ldots, n$ it holds $0 \leq \mu_{f^{+}}\left(A_{i}\right) \leq c$ and $\mu_{f^{+}}\left(A_{1}\right)=c>0$ or $0 \leq \tilde{\mu}_{f^{+}}\left(A_{i}\right) \leq c$ and $\tilde{\mu}_{f}\left(A_{1}\right)=c>0$. By Proposition 1, in both subcases we get $B C h(\varphi(f), \mathbf{m}) \geq 0$ and $B C h(f, \mathbf{m}) \geq 0$ and if we suppose $B C h(\varphi(f), \mathbf{m})=0$, then it follows $B C h(f, \mathbf{m})=0$.

Now, suppose that $B C h(\varphi(f), \mathbf{m})>0$ and for all $i=1, \ldots, n$ it holds $0 \leq \mu_{f^{+}}\left(A_{i}\right) \leq c$ and $\mu_{f^{+}}\left(A_{1}\right)=c>0$. By Proposition 1 we have $\varphi\left(\frac{1}{c}|B C h(f, \mathbf{m})|\right)=\varphi\left(\frac{1}{c} B C h(f, \mathbf{m})\right)$

$$
\begin{aligned}
& =\varphi\left(\frac{1}{c} \sum_{i=1}^{n}\left|f_{\alpha(i)}\right| \cdot\left(\mu_{f^{+}}\left(A_{i}\right)-\mu_{f^{+}}\left(A_{i+1}\right)\right)\right) \\
& =\varphi\left(\sum_{i=1}^{n}\left|f_{\alpha(i)}\right| \cdot\left(\frac{\mu_{f^{+}}\left(A_{i}\right)}{c}-\frac{\mu_{f^{+}}\left(A_{i+1}\right)}{c}\right)\right) \\
& \leq \sum_{i=1}^{n} \varphi\left(\left|f_{\alpha(i)}\right|\right) \cdot\left(\frac{\mu_{f^{+}}\left(A_{i}\right)}{c}-\frac{\mu_{f^{+}}\left(A_{i+1}\right)}{c}\right) \\
& =\frac{1}{c} \sum_{i=1}^{n}\left|\varphi\left(f_{\alpha(i)}\right)\right| \cdot\left(\mu_{f^{+}}\left(A_{i}\right)-\mu_{f^{+}}\left(A_{i+1}\right)\right) \\
& =\frac{1}{c} \cdot \operatorname{BCh}(\varphi(f), \mathbf{m}) \\
& =\frac{1}{c} \cdot|B C h(\varphi(f), \mathbf{m})|,
\end{aligned}
$$

where the inequality holds based on the well-known Jensen's type inequality for ordered weighted means with real weights $p_{i}$, such that $0 \leq \sum_{i=1}^{k} p_{i} \leq 1$, for all $k=1, \ldots, n-1$ and $\sum_{i=1}^{n} p_{i}=1$ (see, e.g. $[3,17,23,30]$ ).

Analogously we obtain the claim in the second subcase, i.e. if for all $i=1, \ldots, n$ it holds $0 \leq \tilde{\mu}_{f^{+}}\left(A_{i}\right) \leq c$ and $\tilde{\mu}_{f^{+}}\left(A_{1}\right)=c>0$.

Case II For all $i=1, \ldots, n$ it holds $-c \leq \mu_{f^{+}}\left(A_{i}\right) \leq 0$ and $\mu_{f^{+}}\left(A_{1}\right)=-c<0$ or $-c \leq \tilde{\mu}_{f^{+}}\left(A_{i}\right) \leq 0$ and $\tilde{\mu}_{f^{+}}\left(A_{1}\right)=-c<0$. In both subcases, by Proposition 1 , we obtain $B C h(\varphi(f), \mathbf{m}) \leq 0$ and $B C h(f, \mathbf{m}) \leq 0$. Therefore

$$
|B C h(\varphi(f), \mathbf{m})|=-B C h(\varphi(f), \mathbf{m}) \text {, and }|B C h(f, \mathbf{m})|=-B C h(f, \mathbf{m}) .
$$

Moreover, if $B C h(\varphi(f), \mathbf{m})=0$, then $B C h(f, \mathbf{m})=0$.

Further, suppose $B C h(\varphi(f), \mathbf{m})<0$ and for all $i=1, \ldots, n$ it holds $-c \leq$ $\mu_{f^{+}}\left(A_{i}\right) \leq 0$ and $\mu_{f^{+}}\left(A_{1}\right)=-c<0$. By (8) it follows that for all $i=1, \ldots, n$ it holds $0 \leq \tilde{\hat{\mu}}_{(-f)^{+}}\left(A_{i}\right) \leq c$ and $\tilde{\hat{\mu}}_{(-f)^{+}}\left(A_{1}\right)=c>0$. Now, from Case I we obtain

$$
\varphi\left(\frac{1}{c} \cdot|B C h(-f, \widehat{\mathbf{m}})|\right) \leq \frac{1}{c} \cdot|B C h(\varphi(-f), \widehat{\mathbf{m}})| .
$$


Finally, by Proposition 2 and the previous inequality, we get

$$
\begin{aligned}
\varphi\left(\frac{1}{C}|B C h(f, \mathbf{m})|\right) & =\varphi\left(\frac{1}{c} \cdot B C h(-f, \widehat{\mathbf{m}})\right) \\
& =\varphi\left(\frac{1}{c} \cdot|B C h(-f, \widehat{\mathbf{m}})|\right) \\
& \leq \frac{1}{c} \cdot|B C h(\varphi(-f), \widehat{\mathbf{m}})| \\
& =\frac{1}{c} \cdot|B C h(\varphi(f), \mathbf{m})| .
\end{aligned}
$$

Analogously we obtain the claim in the second subcase, i.e. if for all $i=1, \ldots, n$ it holds $-c \leq \tilde{\mu}_{f^{+}}\left(A_{i}\right) \leq 0$ and $\tilde{\mu}_{f^{+}}\left(A_{1}\right)=-c<0$.

We have the next consequence of the previous theorem.

Corollary 3 Let $\varphi:[-1,1] \rightarrow[-1,1], \varphi(1)=1$, be an odd and strictly increasing function, such that it is concave on $[0,1]$, and $c>0$. Let $f \in \mathcal{S}$ and $\mathbf{m} \in \mathcal{M}$. If there exists a permutation $\alpha$ of indexes such that $\left|f_{\alpha(i)}\right| \leq\left|f_{\alpha(j)}\right|$, for all $i \leq j$, and for all $i, j=1, \ldots, n$ it holds $\mu_{f^{+}}\left(A_{i}\right) \mu_{f^{+}}\left(A_{j}\right) \geq 0,\left|\mu_{f^{+}}\left(A_{i}\right)\right| \leq c$ and $\left|\mu_{f^{+}}\left(A_{1}\right)\right|=$ $c \quad$ or $\quad \tilde{\mu}_{f^{+}}\left(A_{i}\right) \tilde{\mu}_{f^{+}}\left(A_{j}\right) \geq 0, \quad\left|\tilde{\mu}_{f^{+}}\left(A_{i}\right)\right| \leq c \quad$ and $\quad\left|\tilde{\mu}_{f^{+}}\left(A_{1}\right)\right|=c$, where $A_{i}=\left\{x_{\alpha(i)}, \ldots, x_{\alpha(n)}\right\}$, then

$$
\varphi\left(\frac{1}{c} \cdot|B C h(f, \mathbf{m})|\right) \geq \frac{1}{c} \cdot|B C h(\varphi(f), \mathbf{m})| .
$$

Proof. The claim follows from the fact that $\varphi^{-1}$ is odd and strictly increasing on $[-1,1]$ and convex on $[0,1]$.

The last example shows that (13) holds even if the condition $\varphi(1)=1$ is omitted. Notice that if $\varphi$ is odd, strictly decreasing on $[-1,1], \varphi(1)=-1$, and concave (convex) on $[0,1]$, then $\varphi(|x|) \in[-1,0]$, and $-\varphi\left(-\varphi^{-1}\right.$ resp.) is odd, strictly increasing on $[-1,1]$ and convex on $[0,1]$. Therefore, as consequences of Theorem 3, we have:

$$
\varphi\left(\frac{1}{c} \cdot|B C h(f, \mathbf{m})|\right) \underset{(\leq)}{\geq}-\frac{1}{c} \cdot|B C h(\varphi(-f), \mathbf{m})| .
$$

Example 5 ([17]) Let $X=\left\{x_{1}, x_{2}, x_{3}, x_{4}\right\}$ and $\varphi(x)=\operatorname{sign}(x)\left(1-e^{-|x|}\right)$, $x \in[-1,1]$, concave on $[0,1]$. Let $f \in$ be $\mathcal{S}$ defined by $f\left(x_{1}\right)=-0.2$, $f\left(x_{2}\right)=0.4, f\left(x_{3}\right)=-0.6$, and $f\left(x_{4}\right)=0.8$. We have $\left|f\left(x_{1}\right)\right| \leq\left|f\left(x_{2}\right)\right| \leq$ $\left|f\left(x_{3}\right)\right| \leq\left|f\left(x_{4}\right)\right|$, and corresponding $A_{i}$ and $\mu_{f^{+}}\left(A_{i}\right)$ are given in Table 1.

Table 1

Corresponding $A_{i}$ and $\mu_{f^{+}}\left(A_{i}\right)$ for $\alpha=(1,2,3,4)$

\begin{tabular}{|c|c|c|c|c|}
\hline$\alpha(i)$ & 1 & 2 & 3 & 4 \\
\hline$\left|f_{\alpha(i)}\right|$ & 0.2 & 0.4 & 0.6 & 0.8 \\
\hline$A_{i}$ & $X$ & $\left\{x_{2}, x_{3}, x_{4}\right\}$ & $\left\{x_{3}, x_{4}\right\}$ & $\left\{x_{4}\right\}$ \\
\hline$\mu_{f}+\left(A_{i}\right)$ & 0.6 & 0.6 & 0.2 & 0.4 \\
\hline
\end{tabular}


We compute:

$$
\begin{aligned}
\varphi\left(\frac{1}{0.6} \cdot B C h\right. & (f, \mathbf{m}))=\varphi\left(\frac{1}{0.6} \cdot((0.2-0) \cdot 0.6+(0.4-0.2) \cdot 0.6+\right. \\
+ & (0.6-0.4) \cdot 0.2+(0.8-0.6) \cdot 0.4)) \\
& =1-e^{-\frac{0.36}{0.6}} \approx 0.45119 ; \\
\frac{1}{0.6} \cdot B C h(\varphi(f), \mathbf{m}) & =\frac{1}{0.6} \cdot((\varphi(0.2)-\varphi(0)) \cdot 0.6+(\varphi(0.4)-\varphi(0.2)) \cdot 0.6+ \\
+ & (\varphi(0.6)-\varphi(0.4)) \cdot 0.2+(\varphi(0.8)-\varphi(0.6)) \cdot 0.4) \\
& =\frac{1}{0.6} \cdot\left(\left(1-e^{-0.2}\right) \cdot 0.6+\left(e^{-0.2}-e^{-0.4}\right) \cdot 0.6+\right. \\
+ & \left.\left(e^{-0.4}-e^{-0.6}\right) \cdot 0.2+\left(e^{-0.6}-e^{-0.8}\right) \cdot 0.4\right) \approx 0.43650 .
\end{aligned}
$$

Therefore, $\varphi\left(\frac{1}{0.6} \cdot \mid B C h(f, \mathbf{m} \mid)\right) \geq \frac{1}{0.6} \cdot|B C h(\varphi(f, \mathbf{m}))|$, i.e. the inequality (13) is satisfied.

\section{Conclusions}

The Jensen type inequality for the discrete bipolar Choquet, Sugeno and Shilkret integrals with respect to normalized bi-capacities have been proven. The main results are in accordance with the Jensen type inequality for the bipolar pseudointegrals presented in [30] and with the corresponding inequality for CPT-like integrals studied in the framework of principles for premium calculation in [16]. In the case of non-negative functions, the obtained results are in accordance with the corresponding inequality for the Sugeno integral obtained in [21] and the inequality for Choquet integral studied in $[15,16]$. For the first time in the literature dealing with bipolar integrals, the Jensen type of inequality was considered in the recent paper [30]. Therefore, in the future work the application of the obtained results in decision-making problems will be studied.

\section{Acknowledgment}

The authors acknowledge the financial support of the Ministry of Education, Science and Technological Development of the Republic of Serbia (the first author: project "Innovative scientific and artistic research from the FTS (activity) domain" Grant No. 451-03-68/2020-14/200156, the second author: Grant No. 45103-9/2021-14/ 200125).

\section{References}

[1] S. Abbaszadeh, M. E. Gordji, E. Pap, A. Szakál: Jensen-type inequalities for Sugeno integral, Inf. Sci. 376 (2017) 148-157

[2] H. Agahi, R. Mesiar, A. Babakhani: Generalized expectation with general kernels on g-semirings and its applications, RACSAM 111 (2017) 863-875

[3] P. S. Bullen: Accentuate the negative, Math. Bohem. 134 (2009) 427-446 
[4] D. Denneberg: Non-Additive Measure and Integral, Kluwer Academic, Dordrecht, 1994

[5] G. Choquet: Theory of capacities, Ann. Inst. Fourier 5 (1953/54) 131-295

[6] M. Grabisch: Set Functions, Games and Capacities in Decision Making, Springer, Cham, 2016

[7] M. Grabisch, C. Labreuche: Bi-capacities, Part I: definition, Möbius transform and interaction, Fuzzy Sets Syst. 151 (2005) 211-236

[8] M. Grabisch, C. Labreuche: Bi-capacities, Part II: the Choquet integral, Fuzzy Sets Syst. 151 (2005) 237-259

[9] M. Grabisch, J. L. Marichal, R. Mesiar, Pap, E.: Aggregation Functions (Encyclopedia of Mathematics and its Applications) Cambridge University Press 2009

[10] S. Greco, B. Matarazzo, R. Slowinski: Bipolar Sugeno and Choquet integrals, in: G. Pasi B. De Baets, J. Fodor (Eds.), Workshop on Information Systems (EURO-FUSE 2002), Varenna, Italy (2002) 191-196

[11] S. Greco, R. Mesiar, F. Rindone: Discrete bipolar universal integrals, Fuzzy Sets Syst. 252 (2014) 55-65

[12] S. Greco, F. Rindone: Bipolar fuzzy integrals, Fuzzy Sets Syst. 220 (2013) 21-33

[13] M. Kaluszka, A. Okolewski, M. Boczek: On the Jensen type inequality for generalized Sugeno integral, Inf. Sci. 266 (2014) 140-147

[14] P. Karczmarek, A. Kiersztyn, W. Pedrycz: Generalized Choquet integral for face recognition, Int. J. Fuzzy Syst. 20 (2018) 1047-1055

[15] R. Mesiar, J. Li, E. Pap: The Choquet integral as Lebesgue integral and related inequalities, Kybernetika 46 (2010) 1098-1107

[16] B. Mihailović, E. Pap, M. Štrboja, A. Simićević: A unified approach to the monotone integral-based premium principles under the CPT theory, Fuzzy Sets and Syst. 398 (2020) 78-97

[17] B. Mihailović, M. Štrboja, M. Todorov: The bipolar Choquet g-integrals, in: Proceedings of $17^{\text {th }}$ IEEE International Symposium on Intelligent Systems and Informatics, Subotica (2019) 173-178

[18] E. Pap: Null-Additive Set Functions, Kluwer Academic Publishers, Dordrecht- Boston-London, 1995

[19] E. Pap (ed.): Handbook of Measure Theory, Elsevier Science, Amsterdam, 2002

[20] E. Pap, M. Štrboja: Generalization of the Jensen inequality for pseudointegral, Inf. Sci. 180 (2010) 543-548 
[21] H. Román-Flores, A. Franulič, Chalko-Cano: A Jensen type inequality for fuzzy integrals, Inf. Sci. 177 (2007) 3192-3201

[22] N. Shilkret: Maxitive measure and integration, Indag. Math. 74 (1971) 109116

[23] J. F. Steffensen: On certain inequalities and methods of approximation, J. Inst. Actuar. 51 (1919) 274-297

[24] M. Štrboja, B. Mihailović: Generalized Minkowski type inequality for pseudo-integral, Math. Slovaca 71(1) (2021) 57-74

[25] M. Štrboja, E. Pap, B. Mihailović: Discrete bipolar pseudo-integrals, Inf. Sci. 468 (2018) 72-88

[26] M. Sugeno: Theory of Fuzzy Integrals and its Applications, Ph.D. Thesis, Tokyo Institute of Technology, 1974

[27] M. Sugeno, T. Murofushi: Pseudo-additive measures and integrals, J. Math. Anal. Appl. 122 (1987) 197-222

[28] M. Todorov, M. Štrboja, B. Mihailović: Bi-capacities based pan-integral, in: Proceedings of $16^{\text {th }}$ IEEE International Symposium on Intelligent Systems and Informatics, Subotica, Serbia (2018) 301-304

[29] M. Todorov, M. Štrboja, B. Mihailović: Jensen type inequality for the bipolar Shilkret and Sugeno integrals, in: Proceedings of $17^{\text {th }}$ IEEE International Symposium on Intelligent Systems and Informatics, Subotica (2019) 179-184

[30] M. Todorov, M. Štrboja, E. Pap, B. Mihailović: Jensen type inequality for the bipolar pseudo-integrals, Fuzzy Sets and Syst. 379 (2020) 82-101

[31] A. Tversky, D. Kahmenam: Advances in prospect theory. Cumulative representation of uncertanity, J. of Risk and Uncertanity 5 (1992) 297-323 